\title{
Kids' Survivability as Affected by Their Body Weight, Blood Biochemical Indices and Maternal and Kids' Behavior in Baladi and Shami Goats under Semi-Arid Condition
}

\author{
Nagy H. Ibrahim ${ }^{1}$, Mohamed T. Badawy ${ }^{2}$; Ibrahim A. Zakzouk ${ }^{2}$ and Fawzy E. Younis ${ }^{2}$ \\ ${ }^{1}$ Animal and Poultry Production Department, Faculty of Agriculture, Beni-Suef University, Beni-Suef, Egypt. \\ ${ }^{2}$ Animal and Poultry Physiology Department, Desert Research Center, Cairo, Egypt \\ *Corresponding author`s Email: nagymetwally@agr.bsu.edu.eg; (DORCID: 0000-0001-9214-1860
}

\begin{abstract}
The present study was conducted to investigate the effect of body weight, blood biochemical parameters and post parturient behavioral activities of goats and their kids on kids' mortality rate in Baladi and Shami breeds during neonatal period. Twenty-five adults does of each breed (average age: 18 months old) were selected during breeding season. All female goats were estrus synchronized and naturally mated. After parturition, one hundred and one kids (39 Baladi and 62 Shami) were followed for up to 30 days of their age. The overall mean birth weights of female kids of Baladi and Shami goats were 2.47 and $2.81 \mathrm{Kg}$, respectively. For male kids, birth weights were 2.43 and 2.47 $\mathrm{kg}$, respectively. There was no significant difference in average daily gain ( $\mathrm{g} /$ day) between Baladi and Shami kids during the first 30 days of age. Male kids recorded higher mortality rate than female kids. The percent of death for male and female kids were $87.50 \%$ and $36.84 \%$ in Shami while, were $33.33 \%$ and $14.28 \%$ in Baladi respectively. However, death stopped in Baladi kids after 14 days, but continued in Shami kids to 28 days post-partum. Present data revealed that goat breed and neonatal period showed a significant effect on urea concentration and alkaline phosphatase (ALP) and insignificant effect on creatinine concentration, alanine transferase (ALT), gamma glutamyl transferase (GGT), total lipids, cholesterol and triglycerides in Baladi and Shami Kids. While aspartate transferase (AST), were not affected by goat breed and was significantly affected by both age of birth and interactions. Baladi breed showed significantly better maternal activity than Shami does as they spend lesser time to concern their newly born kids. Baladi kids had more strong behavior towards their dams when compared to Shami ones. It was concluded that body weight, blood biochemical parameters and Maternal and kid's behavior had notable effect on kid's survivability. Our results might declare superiority of Baladi kids than Shami ones which reflected on the significant reduction of mortality rate in Baladi kids as compared to Shami ones.
\end{abstract}

Key words: Body weight, Goat, Kids behavior, Maternal behavior, Offspring survival

\section{INTRODUCTION}

Goats (Capra hircus) have a variety of functions and display a high ability to adapt and maintain themselves in harsh environment (Girma et al., 2011). The population of Egyptian goats is estimated by 4.2 million heads (FAOSTAT, 2012). This population is distributed across the country, especially in the Nile valley and delta region followed by the north-western coastal region and at oases (Galal et al., 2005). However, in Sinai goat population is almost three folds of that of sheep CAPMAS (2016). Kids' mortality has a direct effect on genetic progress by its effect on selection pressure. Generally, greater kids' mortality happens at birth and from birth to weaning, while mortality is comparatively low from weaning to breeding age in several production systems (Donkin and Boyazoglu, 2004). Several factors had been reported in the literature that affected mortality rate in goat kids such as kind of birth, sex, weight of birth, parity, kidding season, age of the kids and kidding year (Hailu et al., 2006; Mtenga et al.,2008 and Khalil et al., 2018).

Awemu et al. (2002) and EL-Tonsy et al., (2018) reported that the kid's mortality was significantly affected by birth weight. Madibela et al. (2002) stated that persistence rates were similar (93.9 versus $91.9 \%$ ) between singles and multiples in Tswana goats. Steve and Marco (2001) and Snyman (2010) stated that survival to weaning of goat kids appeared higher for male gender than for females. However, Ingo (2002) reported that sex is not significant factor in kid survival.

The haemato-biochemical profiles can be previously reported to assess the immunity in offspring (Al-Seaf and AlHarbi, 2012). Gwaze et al. (2012) showed that there was a negative relationship $(\mathrm{P}<0.05)$ between age and ALP, while there was no relationship $(\mathrm{P}>0.05)$ between age and selected liver enzymes. Also, Herosimczyk et al. (2011) reported that the decrease in plasma creatinine concentrations after $14^{\text {th }}$ day of age is may be associated with increased glomerular filtration rate in calves.

In mammals, maternal behavior (nursing of the infant and lactation) represent important behavioral and energy investments indispensable for the successful rearing of the offspring (Nowak et al., 2000). However, the degree to which maternal recognition contributes to preferential care and survival of the progeny may vary, depending on the type of mother-young relationships (Martínez et al., 2009). Vulnerable kids physically, especially kids resulting from mothers 
who did not receive good nutrition during pregnancy, have mortality rates higher, as they fail to show the behavior of survival such as searching for nipples and feeding the same most powerful child force of dams that feed well (Browning et al., 2011). Disturbance in maternal care, maternal selectivity, or mutual recognition may have adverse effects on the survival of the young during the first week of life (Poindron et al., 2007; Nowak et al., 2007). In goats, immediately after birth, the mother goats started licking their kids and allowing the kids to breastfeed are the first signs of a mother's behavior (Kaymakci, 2013). Dam behaviors help to clean and dry their offspring from fetal membranes, stimulate the newborn to seek teat and form an exclusive and selective attachment to her own offspring (Nowak et al., 2000; Dwyer, 2009).

The present study was undertaken to investigate the role of birth weight, biochemical parameters, sex, maternal behavior and kid's behavior in kids' mortality rate in both Baladi and Shami goat under semi-arid conditions.

\section{MATERIALS AND METHODS}

\section{Experimental region}

The present study was carried out at Ras-Sudr Research Station (located in190 km East of Cairo) that belongs to the Desert Research Center (DRC), South Sinai Governorate, Egypt. The prevailing climate is characterized by long hot summer, short winter, low rainfall and high evaporation intensities. However, winter nights might be so cold. The experiment was conducted during one month from February to March 2016.

\section{Ethical approval}

This experiment was performed according to all ethics and animal rights regulated by desert research centre, Egypt. As much as this work had considering all rules and regulations in conformity with the European union directive for the protection of experimental animals (2010/63/EU).

\section{Animals}

Twenty-five Baladi does (averaged $21.12 \mathrm{~kg} \mathrm{LBW}$ ) and 25 Shami does (averaged $35.78 \mathrm{~kg} \mathrm{LBW}$ ) all averaged 18 months old, were used in this study to evaluate kids' mortality rate and its reasons. All does were clinically examined. Baladi goats breed was well-adapted to the environmental conditions in North and South Sinai in Egypt, while Shami goat breed was originated in Syria and imported to North Sinai.

\section{Feeding and management}

All goats were housed in semi open shaded pens and were fed uniformly twice a day (08:00 and 14:00 h) on Berseem hay and concentrate feed mixture to cover their nutrient requirements during different physiological status according to Kearl (1982). The chemical compositions of feed stuffs were determined according to AOAC (1990) and showed in table 1 . The two groups were fed these diets for an adaptation period of 21days before mating season and all over the pregnancy period and after parturition till one month after birth. During the trial, all animals drank 3 times a day (08:00, 14:00 and 20:00 h). Chemical composition of drinking water is showed in table 2. All animals were kept under natural photoperiod and ambient temperature. Vaccination for all goats Before the mating season against the main prevalent epidemic diseases, internal and external parasites were controlled in a timely manner in a good way.

Table 1. Chemical composition of experimental feed stuffs (as percentage of dry matter)

\begin{tabular}{lccccccc}
\hline Ingredients & DM & OM & CP & EE & CF & NFE & Ash \\
\hline Berseem hay (\%) & 85.1 & 86.85 & 12.09 & 1.36 & 27.57 & 45.83 & 13.15 \\
Concentrate feed mixture (\%) & 91.42 & 88.61 & 15.61 & 3.01 & 16.33 & 53.66 & 11.39 \\
\hline
\end{tabular}

DM: dry matter; OM: organic matter; CP: Crude protein; EE: ether extract; CF: crude fiber; NFE: Nitrogen free extract

Table 2. Chemical composition of tap water used in the experiment

\begin{tabular}{lccccc}
\hline Items & TDS & $\mathbf{N a}^{+}$ & $\mathbf{K}^{+}$ & $\mathbf{M g}^{+}$ & $\mathbf{C L}^{-}$ \\
\hline Tap water $(\mathbf{p p m})$ & 600 & 145.2 & 9.09 & 15.3 & 179.88
\end{tabular}

TDS: Total dissolved salts

\section{Live body weight}

All kids in both breeds were weighed immediately after birth and each week then after to the end of the experiment. Weighing was performed early in the morning before suckling. Electronic balance was used for measuring weights of kids to the nearest $0.01 \mathrm{~kg}$.

\section{Blood sampling}

Five $\mathrm{ml}$ of blood were collected from the outer jugular vein of Shami and Baladi kids in vacuum tubes containing heparin once a week at the same time in the morning $(8 \mathrm{am})$ during the first month after birth. The first sampling in the 
lambs was performed immediately after birth. Plasma was obtained for chemical analysis by centrifugation of blood at $3000 \mathrm{rpm}$ then preserved at $-20{ }^{\circ} \mathrm{C}$. Biochemical analyses including plasma alanine transferase (ALT), aspartate transferase (AST), alkaline phosphatase (ALP), gamma glutamyl transferase (GGT), urea, creatinine, total lipids, cholesterol and triglycerides assays were recorded in plasma using kits supplemented by Diamond Company.

\section{Behavioral studies}

For behavior observations, does and their kids were kept in open housing pen (observation pen) with dimensions of $6 \mathrm{~m} \mathrm{x} 6 \mathrm{~m}$ and a floor made from cement and padded with rice straw. Does were entered in observation pens with their kids and were observed for 24 hours. All animals (dams and kids) were identified by painting their sides with spray by clear number for this study. Behavioral activities were recorded with eye observation.

\section{Does behavior criteria}

The following criteria were recorded for all does during post-partum till the first $24 \mathrm{~h}$ of birth:

DS: Does stood after delivery (sec).

FAK: First attention to kid (min)

DSL: Does started licking (min)

S/L: Sniffing/ licking during the first two hrs. after birth.

\section{Kids' behavior criteria}

All offspring were observed from parturition up to 24 hours of their age to assess the following patterns:

\section{Standing attempts including}

- First standing attempt (min)

- First success standing (min)

- Time standing during the first two hours after birth (min)

\section{Suckling attempts including}

- First attempted of suckling (min)

- Kids starting suckling (min)

- Duration of first suckling (min)

- Total time of suckling during the first two hours after birth (min)

- Suckling frequency during the first two hours after birth (bouts)

\section{Statistical analysis}

Results were presented as Mean \pm SE. A two-way analysis of variance (ANOVA) considering repeated measurements; was used to determine statistical differences between mean values of the studied parameters during the first month postpartum. Data were analyzed by the least square of analysis variance using the general linear model procedure (SAS, 2004). Duncan's New Multiple Range test (Duncan, 1955) separated differences (P<0.05) among treatment means \{Goat breed (Baladi and Shami); Neonatal period (Immediately, 7-day, 14-day, 21-day, 28-day after birth)\}.

\section{RESULTS AND DISCUSSION}

\section{Mortality rate of Baladi and Shami goat kids during neonatal period}

As presented in table 3, one hundred and one kids (39 Baladi and 62 Shami) were obtained and followed for up to 30 days of their age. Forty-four (9 Baladi and 35 Shami) died before they achieved this age. The age of kids in weeks had a significant impact $(\mathrm{P}<0.05)$ on the death of kids. The 44 dead kid were distributed as follows; 21 (47.72\%) occurred within the first week of their age and then death cases reduced significantly during the following 3 consequent weeks $(29.54,13.63$ and $9.09 \%$, respectively). The overall mortality rate in this study was also significantly affected by the breed that recorded $23.07 \%$ for Baladi and $56.45 \%$ for Shami kids during neonatal period. Sex had a significant effect on mortality rate. Male kids recorded higher mortality rate than females $(33.33 \%$ versus $14.28 \%$, respectively for Baladi kids and $87.50 \%$ versus $36.84 \%$ for Shami kids, respectively).

Present results were totally in agreement with Aganga et al. (2005) and Hailu et al. (2006) as they recorded higher mortality rates for male kids compared to females. On the other hand, Petros et al. (2014) found insignificant effect of sex on pre-weaning mortality, while Debele et al. (2011) recorded higher mortality rates in females than males' kids in Arsi-Bale kids maintained in a similar environment. This might reflect higher immunity of female as stated by Tambuwal et al. (2002) and Ashour et al. (2015) who found in goats a higher leucocyte counts in females than in males.

The results of present study indicated that 13 of Baladi dams gave one kid, 9 gave twins kids and 3 gave triple kids. For Shami dams, the corresponding numbers were 0, 13 and 12, respectively. Mortality tended to increase with a larger size litter where single kids had always a higher birth weight hence higher survival rate than twins and triplets (Awemu et al., 1999). Nevertheless, Madibela et al. (2002) found that survivability rates were similar (91.9 versus 93.9\%) 
between manifold and singles in Tswana goats. They added that survival rates and growth rates can be improved by supplementation. In the present study, kids' mortality occurred in Baladi during the first two weeks, while continued in Shami kids all over the first month with different percentage per week.

Table 3. Means of twinning and mortality rates of Baladi and Shami kids during neonatal

\begin{tabular}{|c|c|c|c|c|c|c|c|c|c|c|c|c|c|c|}
\hline \multirow{3}{*}{ Breed } & \multirow{3}{*}{ Sex } & \multirow{3}{*}{ 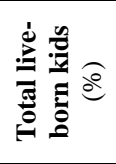 } & \multirow{3}{*}{$\begin{array}{c}\text { Sex } \\
\text { ratio }\end{array}$} & \multirow{3}{*}{ 莦唱 } & \multicolumn{8}{|c|}{$\begin{array}{l}\text { kids die during neonatal period as a percentage of total live-born } \\
\text { kids }\end{array}$} & \multirow{2}{*}{\multicolumn{2}{|c|}{$\begin{array}{l}\text { Total mortality } \\
\quad \text { rate }(\%)\end{array}$}} \\
\hline & & & & & \multicolumn{2}{|c|}{$1^{\text {st }}$ week } & \multicolumn{2}{|c|}{$2^{\text {nd }}$ week } & \multicolumn{2}{|c|}{$3^{\text {rd }}$ week } & \multicolumn{2}{|c|}{$4^{\text {th }}$ week } & & \\
\hline & & & & & $\mathrm{N}$ & $\%$ & $\mathrm{~N}$ & $\%$ & $\mathrm{~N}$ & $\%$ & $\mathrm{~N}$ & $\%$ & $\mathrm{~N}$ & $\%$ \\
\hline \multirow{3}{*}{ Baladi } & Female & 21 & 53.85 & & 2 & 11.11 & 1 & 5.56 & 0 & 0.0 & 0 & 0.0 & 3 & 14.28 \\
\hline & Male & 18 & 46.15 & 156 & 4 & 19.05 & 2 & 9.52 & 0 & 0.0 & 0 & 0.0 & 6 & 33.33 \\
\hline & Total & 39 & 100 & & 6 & 15.38 & 3 & 7.69 & 0 & 0.0 & 0 & 0.0 & 9 & 23.07 \\
\hline \multirow{3}{*}{ Shami } & Female & 38 & 61.29 & & 7 & 18.42 & 4 & 10.52 & 2 & 5.26 & 1 & 2.63 & 14 & 36.84 \\
\hline & Male & 24 & 38.71 & 248 & 8 & 33.33 & 6 & 25.00 & 4 & 16.67 & 3 & 12.50 & 21 & 87.50 \\
\hline & Total & 62 & 100.0 & & 15 & 24.19 & 10 & 16.13 & 6 & 9.78 & 4 & 6.45 & 35 & 56.45 \\
\hline
\end{tabular}

N: number of kids

\section{Body weight changes and growth rates in kids during neonatal period}

The body weight results of Baladi and Shami kids are illustrated in tables 4 and 5. The overall mean birth weights of female kids of Baladi and Shami goats were 2.47 and $2.81 \mathrm{Kg}$ with significant differences $(\mathrm{P}<0.01)$. For male kids, birth weights were 2.43 and $2.47 \mathrm{~kg}$, respectively without significant differences. Male kids' birth weight was significantly lower than female kids. These results were contrary to several reports recording higher birth weight of male than female kids (Gubartalla et al., 2002; ELimam et al.,2007; Bushara et al., 2013). On the other hand, Hanford et al. (2006) recoded that there was no significant change in birth weight due to kids' sex. These differences between author's results might be due to breed differences and management. Baladi male kids showed the lowest birth weight $(2.43 \mathrm{~kg})$ and the highest percentage mortality rate $(66.66 \%)$. Kid's birth weight was found to have a significant effect on kids' mortality (Awemu et al., 2002 and Debele et al., 2011) and final economic benefit (Portolano et al., 2002 and Hanford et al., 2006). Mtenga et al. (1992) reported that the lowest pre-weaning mortality rate occurred in kids with a birth weight greater than $2.6 \mathrm{~kg}$.

The rate of change in live body weight (LBW) for Baladi and Shami was respectively 29.99 versus $33.18 \%$ for females, while was 31.35 versus $26.73 \%$ for males, with the differences being significant $(\mathrm{P}<0.01)$. However, there was no significant change in average daily gain (ADG) between Baladi and Shami kids during neonatal period. In agreement, Khazaal (2009) recorded that ADG of kids was similar between Saanen and Shami during the first three months of age (suckling period). It was apparent that Shami female kids had the highest birth weight, growth rate and final LBW. Baladi male kids that could stay alive compensated the lower birth weight by high relative growth rate and attained the same final LBW as Shami males. The data concerning body weight changes of kids that died then after are presented in table 6. The defects in birth weight and growth rated were higher in Shami kids that might give reason to higher mortality rates in this breed.

Table 4. Means of live body weight total gain daily gain and percentage of body weight change of Baladi and Shami female kids during neonatal period

\begin{tabular}{|c|c|c|c|c|c|c|c|c|c|c|}
\hline \multirow{3}{*}{ Parameter } & \multirow{3}{*}{ Breed } & \multicolumn{5}{|c|}{ Neonatal period } & \multirow{3}{*}{ Overall } & \multirow{2}{*}{\multicolumn{3}{|c|}{ \pm Standard error }} \\
\hline & & \multirow{2}{*}{$\begin{array}{c}\text { Immed. } \\
\text { After birth }\end{array}$} & \multirow{2}{*}{$\begin{array}{c}7 \text { d after } \\
\text { birth }\end{array}$} & \multirow{2}{*}{$\begin{array}{c}14 \text { d after } \\
\text { birth }\end{array}$} & \multirow{2}{*}{$\begin{array}{l}21 \text { d after } \\
\text { birth }\end{array}$} & \multirow{2}{*}{$\begin{array}{l}28 \text { d after } \\
\text { birth }\end{array}$} & & & & \\
\hline & & & & & & & & B & $\mathbf{N}$ & $\mathbf{B} \times \mathbf{N}$ \\
\hline \multirow{3}{*}{ BW (kg) } & Baladi & $1.83^{\mathrm{e}}$ & $2.23^{\mathrm{de}}$ & $2.37^{\text {cde }}$ & $2.77^{\text {bcd }}$ & $2.90^{\mathrm{bc}}$ & $2.42^{\mathrm{F}}$ & \multirow{2}{*}{$0.09^{* *}$} & \multirow{2}{*}{$0.12^{* *}$} & \multirow{2}{*}{$0.13^{* *}$} \\
\hline & Shami & $2.09^{\mathrm{de}}$ & $2.32^{\text {cde }}$ & $2.59^{\mathrm{cd}}$ & $3.21^{\mathrm{ab}}$ & $3.71^{\mathrm{a}}$ & $2.78^{\mathrm{E}}$ & & & \\
\hline & Overall & $1.96^{\mathrm{D}}$ & $2.28^{\mathrm{C}}$ & $2.48^{\mathrm{C}}$ & $2.99^{\mathrm{B}}$ & $3.30^{\mathrm{A}}$ & & & & \\
\hline \multirow{3}{*}{ TG (kg) } & Baladi & $0.00^{c}$ & $0.40^{\mathrm{abc}}$ & $0.13^{b c}$ & $0.40^{\mathrm{abc}}$ & $0.12^{\mathrm{bc}}$ & 0.21 & \multirow{2}{*}{$0.09^{\mathrm{NS}}$} & \multirow{2}{*}{$0.11^{* *}$} & \multirow{2}{*}{$0.17^{*}$} \\
\hline & Shami & $0.00^{\mathrm{c}}$ & $0.23^{\mathrm{abc}}$ & $0.23^{\mathrm{abc}}$ & $0.60^{\mathrm{a}}$ & $0.48^{\mathrm{ab}}$ & 0.30 & & & \\
\hline & Overall & $0.00^{\mathrm{C}}$ & $0.31^{\mathrm{AB}}$ & $0.18^{\mathrm{BC}}$ & $0.50^{\mathrm{A}}$ & $0.30^{\mathrm{AB}}$ & & & & \\
\hline \multirow{3}{*}{ DG (g) } & Baladi & $0.00^{c}$ & $57.94^{\mathrm{abc}}$ & $18.71^{\mathrm{bc}}$ & $58.17^{\text {abc }}$ & $18.05^{b c}$ & 30.57 & \multirow{2}{*}{$9.10^{\mathrm{NS}}$} & \multirow{2}{*}{$15.89^{* *}$} & \multirow{2}{*}{$18.20^{*}$} \\
\hline & Shami & $0.00^{c}$ & $32.85^{\mathrm{abc}}$ & $32.85^{\mathrm{abc}}$ & $85.95^{\mathrm{a}}$ & $68.69^{\mathrm{ab}}$ & 44.07 & & & \\
\hline & Overall & $0.00^{\mathrm{C}}$ & $45.39^{\mathrm{AB}}$ & $25.78^{\mathrm{BC}}$ & $72.06^{\mathrm{A}}$ & $43.37^{\mathrm{AB}}$ & & & & \\
\hline \multirow{3}{*}{ BWC (\%) } & Baladi & $0.00^{c}$ & $19.01^{\mathrm{de}}$ & $7.30^{\mathrm{bc}}$ & $17.16^{\mathrm{abc}}$ & $4.89^{\mathrm{bc}}$ & 9.67 & \multirow{2}{*}{$3.89^{\mathrm{NS}}$} & \multirow{2}{*}{$4.71^{*}$} & \multirow{2}{*}{$7.25^{*}$} \\
\hline & Shami & $0.00^{\mathrm{c}}$ & $11.07^{\mathrm{abc}}$ & $10.13^{\mathrm{abc}}$ & $24.50^{\mathrm{a}}$ & $13.37^{\mathrm{a}}$ & 11.81 & & & \\
\hline & Overall & $0.00^{\mathrm{B}}$ & $15.04^{\mathrm{A}}$ & $8.71^{\mathrm{AB}}$ & $20.83^{\mathrm{A}}$ & $9.13^{\mathrm{AB}}$ & & & & \\
\hline
\end{tabular}

B: Breed; N: Neonatal period; LBW: live body weight; TG: total gain; DG: daily gain; BWC\%: percentage of body weight change; A, B, C, D: values with different letters on the same row are significantly different $(\mathrm{P}<0.05)$; E, F: values with different letters on the same column are significantly different $(\mathrm{P}<0.05)$; $\mathrm{a}, \mathrm{b}, \mathrm{c}, \mathrm{d}$, e; values in the experimental sub-groups within certain trait with different superscripts are significantly different $(\mathrm{P}<$ $0.05)$; NS: non-significant, $*: \mathrm{P}<0.05 ; * *: \mathrm{P}<0.01$. 
Table 5. Means of live body weight total gain daily gain and percentage of body weight change of Baladi and Shami male kids during neonatal period.

\begin{tabular}{|c|c|c|c|c|c|c|c|c|c|c|}
\hline \multirow{3}{*}{ Parameter } & \multirow{3}{*}{ Breed } & \multicolumn{5}{|c|}{ Neonatal period } & \multirow{3}{*}{ Overall } & \multirow{2}{*}{\multicolumn{3}{|c|}{ \pm Standard error }} \\
\hline & & \multirow{2}{*}{$\begin{array}{c}\text { Immed. } \\
\text { After birth }\end{array}$} & \multirow{2}{*}{$\begin{array}{c}7 \text { d after } \\
\text { birth }\end{array}$} & \multirow{2}{*}{$\begin{array}{c}14 \mathrm{~d} \text { after } \\
\text { birth }\end{array}$} & \multirow{2}{*}{$\begin{array}{c}21 \text { d after } \\
\text { birth }\end{array}$} & \multirow{2}{*}{$\begin{array}{c}28 \mathrm{~d} \text { after } \\
\text { birth }\end{array}$} & & & & \\
\hline & & & & & & & & $\mathbf{B}$ & $\mathbf{N}$ & $\mathbf{B} \times \mathbf{N}$ \\
\hline \multirow{3}{*}{ BW (kg) } & Baladi & $1.90^{\mathrm{d}}$ & $1.99^{\mathrm{d}}$ & $2.55^{\mathrm{b}}$ & $2.57^{\mathrm{b}}$ & $2.95^{\mathrm{b}}$ & 2.39 & \multirow{2}{*}{$0.03^{\mathrm{NS}}$} & \multirow{2}{*}{$0.06^{*}$} & \multirow{2}{*}{$0.18^{* *}$} \\
\hline & Shami & $1.93^{\mathrm{d}}$ & $2.14^{\text {cd }}$ & $2.55^{\mathrm{b}}$ & $2.62^{\mathrm{b}}$ & $3.18^{\mathrm{a}}$ & 2.48 & & & \\
\hline & Overall & $1.91^{\mathrm{D}}$ & $2.06^{\mathrm{C}}$ & $2.55^{\mathrm{B}}$ & $2.59^{\mathrm{B}}$ & $3.06^{\mathrm{A}}$ & & & & \\
\hline \multirow{3}{*}{ TG (kg) } & Baladi & $0.00^{c}$ & $0.08^{\mathrm{c}}$ & $0.56^{\mathrm{a}}$ & $0.36^{\mathrm{ab}}$ & $0.49^{\mathrm{a}}$ & 0.30 & \multirow{2}{*}{$0.05^{\mathrm{NS}}$} & \multirow{2}{*}{$0.07^{*}$} & \multirow{2}{*}{$0.11^{*}$} \\
\hline & Shami & $0.00^{\mathrm{c}}$ & $0.20^{\mathrm{bc}}$ & $0.39^{\mathrm{ab}}$ & $0.06^{\mathrm{c}}$ & $0.55^{\mathrm{a}}$ & 0.24 & & & \\
\hline & Overall & $0.00^{\mathrm{C}}$ & $0.14^{\mathrm{C}}$ & $0.47^{\mathrm{A}}$ & $0.21^{\mathrm{B}}$ & $0.52^{\mathrm{A}}$ & & & & \\
\hline \multirow{3}{*}{ DG (g) } & Baladi & $0.00^{\mathrm{c}}$ & $12.21^{\mathrm{c}}$ & $81.00^{\mathrm{a}}$ & $51.73^{\mathrm{bc}}$ & $70.31^{\mathrm{a}}$ & 43.05 & \multirow{2}{*}{$0.06^{\mathrm{NS}}$} & \multirow{2}{*}{$7.69^{*}$} & \multirow{2}{*}{$10.87^{*}$} \\
\hline & Shami & $0.00^{\mathrm{c}}$ & $29.57^{\mathrm{c}}$ & $55.86^{\mathrm{ab}}$ & $9.34^{\mathrm{c}}$ & $79.20^{\mathrm{a}}$ & 34.79 & & & \\
\hline & Overall & $0.00^{\mathrm{C}}$ & $20.89^{\mathrm{B}}$ & $68.43^{\mathrm{A}}$ & $30.54^{\mathrm{B}}$ & $74.75^{\mathrm{A}}$ & & & & \\
\hline \multirow{3}{*}{ BWC (\%) } & Baladi & $0.00^{\mathrm{d}}$ & $4.60^{\mathrm{cd}}$ & $27.73^{\mathrm{a}}$ & $16.99^{\mathrm{b}}$ & $21.26^{\mathrm{ab}}$ & 14.12 & \multirow{2}{*}{$1.83^{\mathrm{NS}}$} & \multirow{2}{*}{$3.33^{*}$} & \multirow{2}{*}{$3.17^{*}$} \\
\hline & Shami & $0.00^{\mathrm{d}}$ & $10.53^{\mathrm{cd}}$ & $16.56^{\mathrm{b}}$ & $4.57^{\mathrm{cd}}$ & $21.17^{\mathrm{ab}}$ & 10.57 & & & \\
\hline & Overall & $0.00^{\mathrm{C}}$ & $7.56^{\mathrm{BC}}$ & $22.15^{\mathrm{A}}$ & $10.78^{\mathrm{B}}$ & $21.22^{\mathrm{A}}$ & & & & \\
\hline
\end{tabular}

B: Breed; N: Neonatal period; LBW: live body weight; TG: total gain; DG: daily gain; BWC\%: percentage of body weight change; A, B, C, D: values with different letters on the same row are significantly different $(\mathrm{P}<0.05) ; \mathrm{a}, \mathrm{b}, \mathrm{c}, \mathrm{d}$; values in the experimental sub-groups within certain trait with different superscripts are significantly different $(\mathrm{P}<0.05)$; NS: non-significant; *: $\mathrm{P}<0.05 ; * *: \mathrm{P}<0.01$

Table 6. Live body weight $(\mathrm{kg})$ and percentage change of alive and dead Baladi and Shami kids during neonatal period

\begin{tabular}{|c|c|c|c|c|c|c|c|c|c|c|c|c|c|}
\hline \multirow{3}{*}{ 苞 } & \multirow{3}{*}{ 苍 } & \multirow{3}{*}{ 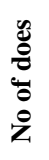 } & \multicolumn{11}{|c|}{ Neonatal period } \\
\hline & & & \multicolumn{6}{|c|}{ Females } & \multicolumn{5}{|c|}{ Male } \\
\hline & & & Case & $\begin{array}{c}\text { 0 } \\
\text { Day }\end{array}$ & $\begin{array}{c}7 \\
\text { day }\end{array}$ & $\begin{array}{c}14 \\
\text { Day }\end{array}$ & $\begin{array}{c}21 \\
\text { day }\end{array}$ & $\begin{array}{c}28 \\
\text { day }\end{array}$ & $\begin{array}{c}\text { 0 } \\
\text { Day }\end{array}$ & $\begin{array}{c}7 \\
\text { day }\end{array}$ & $\begin{array}{c}14 \\
\text { day }\end{array}$ & $\begin{array}{c}21 \\
\text { day }\end{array}$ & $\begin{array}{c}28 \\
\text { Day }\end{array}$ \\
\hline \multirow{9}{*}{ 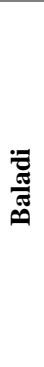 } & \multirow{3}{*}{$\begin{array}{l}\frac{0}{0 D} \\
\stackrel{=}{\sigma}\end{array}$} & \multirow{3}{*}{13} & Alive & 1.83 & 2.23 & 2.37 & 2.77 & 2.90 & 1.90 & 1.99 & 2.55 & 2.57 & 2.95 \\
\hline & & & Dead & 1.55 & 1.61 & 1.68 & . & . & 1.67 & 1.70 & 1.97 & . & . \\
\hline & & & $\%$ change & -15.3 & -27.8 & -29.1 & . & . & -12.1 & -14.6 & -22.7 & . & . \\
\hline & \multirow{3}{*}{$\stackrel{n}{3}$} & \multirow{3}{*}{9} & Alive & 1.74 & 1.89 & 1.95 & 2.10 & 2.90 & 1.79 & 1.90 & 2.23 & 2.30 & 2.41 \\
\hline & & & Dead & 1.35 & 1.39 & 1.44 & . & . & 1.41 & 1.43 & 1.49 & . & . \\
\hline & & & $\%$ change & -22.4 & -26.5 & -26.2 & . & . & -21.8 & -24.7 & -33.2 & . & . \\
\hline & \multirow{3}{*}{$\frac{\frac{0}{0}}{\frac{0}{2}}$} & \multirow{3}{*}{3} & Alive & 1.61 & 1.68 & 1.75 & 1.89 & 2.01 & 1.68 & 1.72 & 1.80 & 1.92 & 2.04 \\
\hline & & & Dead & 1.30 & 1.32 & 1.37 & . & . & 1.35 & 1.38 & 1.40 & . & . \\
\hline & & & $\%$ change & $-19 \%$ & $-21 \%$ & $-21 \%$ & . & . & $-19 \%$ & $-19 \%$ & $-22 \%$ & . & . \\
\hline \multirow{9}{*}{ 离 } & \multirow{3}{*}{$\underset{\stackrel{0}{0 D}}{\Xi}$} & \multirow{3}{*}{0} & Alive & . & . & . & . & . & . & . & . & . & . \\
\hline & & & Dead & . & . & . & . & . & . & . & . & . & . \\
\hline & & & $\%$ change & . & . & . & . & . & . & . & . & . & . \\
\hline & \multirow{3}{*}{$\stackrel{\mathscr{a}}{3}$} & \multirow{3}{*}{13} & Alive & 1.77 & 1.82 & 1.95 & 2.13 & 2.23 & 1.87 & 1.90 & 2.02 & 2.18 & 2.30 \\
\hline & & & Dead & 1.40 & 1.44 & 1.49 & 1.55 & 1.61 & 1.47 & 1.50 & 1.52 & 1.58 & 1.67 \\
\hline & & & $\%$ change & -20.9 & -20.9 & -23.6 & -27.2 & -27.8 & -21.4 & -21.1 & -24.8 & -27.5 & -27.4 \\
\hline & \multirow{3}{*}{$\frac{0}{\frac{0}{2}}$} & \multirow{3}{*}{12} & Alive & 1.70 & 1.78 & 1.86 & 1.96 & 2.14 & 1.75 & 1.81 & 1.99 & 2.11 & 2.33 \\
\hline & & & Dead & 1.35 & 1.38 & 1.43 & 1.48 & 1.55 & 1.40 & 1.44 & 1.49 & 1.55 & 1.63 \\
\hline & & & $\%$ change & -20.6 & -22.5 & -23.1 & -24.5 & -27.6 & -20.0 & -20.4 & -25.1 & -26.5 & -30.0 \\
\hline
\end{tabular}

Physiological changes in hepatic enzyme of Baladi and Shami kids during neonatal period

Enzymes concentrations of alanine transferase (ALT), aspartate transferase (AST), alkaline phosphatase (ALP) and gamma glutamyl transferase (GGT) are those conventionally manipulated for diagnosing hepatic damage. The data presented in table 7 illustrated that goat breed, neonatal period and their interactions $(\mathrm{B} \times \mathrm{N})$ showed insignificant effect on plasma ALT and GGT activities. In disagreement to Njidda et al. (2014) who indicated that there is an influence of age, sex and breed on plasma GGT and ALT activities. On the other hand, present findings revealed that goat breed, neonatal period and their interactions $(\mathrm{B} \times \mathrm{N})$ showed a significant effect on ALP activities in Baladi and Shami Kids. While AST was not affected by goat breed and was significantly affected by both age of birth and interactions $(\mathrm{B} \times \mathrm{N})$. These results are in agreement with Antunovic et al. (2004) who reported a decrease in ALP level with an increase in age of goats. Similar results recorded by Tibbo et al. (2008) and Piccione et al. (2010) who had similar conclusions in ArsiBale goats and Girgentana goats, respectively. Elitok (2012) reported that higher values of ALP were found in the first 40 days of life due to the more intense bone remodeling. The differences were also detected due to both sex and age in accordance with the study of Red Sokoto goats (Tambuwal et al., 2002, Daramola et al., 2005). Smith and Sherman (2009) reported that quality, quantity, and timing of colostrum feeding have an influence on the activity of GGT during the first few days of life. In addition to colostrum intake, the greater activity of ALP in growing goats may be correlated 
to Bone-ALP iso-enzyme. As shown in table 8 generally all liver enzymes (ALT, AST, ALP and GGT) for dead kids in both breeds were decreased as compared to alive ones. The percentages of ALT decreased in Baladi dead kids as compared with a live kids within the 14 days post-partum were between $(-73.49,-73.61$ and $-73.20 \%)$ and (-72.05, 73.54 and $-75.71 \%$ ) for Shami kids through the same period, then increased within the following two weeks to reach (75.91 and $-73.89 \%$ ) in Shami kids, while in AST, ALP and GGT were lower in Baladi dead kids as compared with live kids within the first two weeks post-partum it were between $(-56.69,-65.82$ and $-65.06 \%),(-76.89,-76.19$ and $-74.08 \%)$, $(-54.67,-53.85$ and $-46.58 \%)$ for Baladi dead kids and the percentage in Shami dead kids were $(-64.46,-67.29$ and $68.18 \%),(-76.30,-75.56$ and $-74.75 \%),(-55.70,-52.55$ and $-52.24 \%)$ then, AST increased $(-72.58$ and $-76.56 \%)$, while ALP and GGT decreased in (-74.47 and $-71.37 \%),(-47.58$ and $-39.91 \%)$ respectively in Shami kids within the following two weeks. Decreasing in ALT, AST and ALP activities were higher in Shami kids that might give reason to higher mortality rates in this breed.

Table 7. Hepatic enzyme activity of Baladi and Shami kids during the first month of post-partum

\begin{tabular}{|c|c|c|c|c|c|c|c|c|c|c|}
\hline \multirow{2}{*}{ Parameter } & \multirow[t]{2}{*}{ Breed (B) } & \multicolumn{5}{|c|}{ Neonatal periods $(S)$} & \multirow{2}{*}{$\begin{array}{c}\text { Overall } \\
\text { mean }\end{array}$} & \multicolumn{3}{|c|}{ \pm Standard error } \\
\hline & & 0 day & 7 day & 14 day & 21 day & 28 day & & B & $\mathrm{N}$ & $\mathrm{B} \times \mathrm{N}$ \\
\hline \multirow{3}{*}{$\begin{array}{c}\text { ALT } \\
(\mathbf{I U} / \mathbf{L})\end{array}$} & Baladi & 64.22 & 68.38 & 68.66 & 67.01 & 69.96 & \multirow{3}{*}{$\begin{array}{l}67.65 \\
62.44\end{array}$} & \multirow{3}{*}{$2.84^{\mathrm{NS}}$} & \multirow{3}{*}{$4.10^{\mathrm{NS}}$} & \multirow{3}{*}{$6.36^{\mathrm{NS}}$} \\
\hline & Shami & 53.72 & 56.89 & 66.00 & 68.92 & 66.66 & & & & \\
\hline & Overall mean & 58.97 & 61.05 & 67.33 & 67.96 & 68.31 & & & & \\
\hline \multirow{3}{*}{$\begin{array}{c}\text { AST } \\
(\mathbf{I U} / \mathbf{L})\end{array}$} & Baladi & $35.10^{\mathrm{b}}$ & $44.83^{\mathrm{b}}$ & $46.00^{\mathrm{b}}$ & $43.83^{\mathrm{b}}$ & $46.50^{\mathrm{b}}$ & \multirow{3}{*}{$\begin{array}{l}43.10 \\
43.02\end{array}$} & \multirow{3}{*}{$1.56^{\mathrm{NS}}$} & \multirow{3}{*}{$2.26^{* *}$} & \multirow{3}{*}{$3.50^{* *}$} \\
\hline & Shami & $34.33^{\mathrm{c}}$ & $37.92^{\mathrm{b}}$ & $39.60^{\mathrm{b}}$ & $46.55^{\mathrm{b}}$ & $56.32^{\mathrm{a}}$ & & & & \\
\hline & Overall mean & $34.72^{\mathrm{B}}$ & $41.38^{\mathrm{B}}$ & $42.80^{\mathrm{B}}$ & $45.19^{\mathrm{B}}$ & $51.41^{\mathrm{A}}$ & & & & \\
\hline \multirow{3}{*}{$\begin{array}{c}\text { ALP } \\
(\text { IU/L) }\end{array}$} & Baladi & $82.66^{\mathrm{ab}}$ & $80.66^{\mathrm{bc}}$ & $74.35^{\mathrm{de}}$ & $77.50^{\mathrm{e}}$ & $71.50^{\mathrm{cd}}$ & \multirow{3}{*}{$\begin{array}{l}77.40^{\mathrm{C}} \\
81.41^{\mathrm{D}}\end{array}$} & \multirow{3}{*}{$0.50^{* *}$} & \multirow{3}{*}{$0.73^{* *}$} & \multirow{3}{*}{$1.13^{* *}$} \\
\hline & Shami & $84.44^{\mathrm{a}}$ & $82.66^{\mathrm{a}}$ & $82.88^{\mathrm{ab}}$ & $82.31^{\mathrm{ab}}$ & $74.77^{\mathrm{de}}$ & & & & \\
\hline & Overall mean & $82.66^{\mathrm{A}}$ & $82.55^{\mathrm{A}}$ & $78.61^{\mathrm{B}}$ & $77.30^{\mathrm{B}}$ & $76.13^{\mathrm{B}}$ & & & & \\
\hline \multirow{3}{*}{$\begin{array}{c}\text { GGT } \\
(\text { IU/L) }\end{array}$} & Baladi & 13.26 & 13.48 & 13.33 & 13.40 & 12.33 & \multirow{3}{*}{$\begin{array}{l}13.16 \\
13.13\end{array}$} & \multirow{3}{*}{$0.62^{\mathrm{NS}}$} & \multirow{3}{*}{$0.90^{\mathrm{NS}}$} & \multirow{3}{*}{$1.39^{\mathrm{NS}}$} \\
\hline & Shami & 13.75 & 12.92 & 12.61 & 13.23 & 11.90 & & & & \\
\hline & Overall mean & 13.50 & 13.20 & 13.12 & 13.32 & 12.12 & & & & \\
\hline
\end{tabular}

B: Breed; N: Neonatal period; ALT: alanine transferase; AST: aspartate transferase; ALP: alkaline phosphatase; GGT: gamma glutamyl transferase; A, $\mathrm{B}$ : values with different letters on the same row are significantly different $(\mathrm{P}<0.05)$; $\mathrm{C}$, D: values with different letters on the same column are significantly different $(\mathrm{P}<0.05) ; \mathrm{a}, \mathrm{b}, \mathrm{c}, \mathrm{d}$, e; values in the experimental sub-groups within certain trait with different superscripts are significantly different $(\mathrm{P}<0.05)$; NS: non-significant, $* *: \mathrm{P}<0.01$.

Table 8. Means of liver enzymes and percentage change of alive and dead Baladi and Shami kids during the postnatal period

\begin{tabular}{|c|c|c|c|c|c|c|c|}
\hline \multirow[b]{2}{*}{ Breed } & \multirow[t]{2}{*}{ Parameter } & \multicolumn{6}{|c|}{ The neonatal period (Days) } \\
\hline & & Case & O day & 7 day & 14 day & 21 day & 28 day \\
\hline \multirow{12}{*}{ Baladi } & \multirow{3}{*}{$\begin{array}{c}\text { ALT } \\
(\mathrm{IU} / \mathrm{L})\end{array}$} & Alive & 64.22 & 68.38 & 68.66 & 67.01 & 69.96 \\
\hline & & Dead & 17.02 & 18.04 & 18.40 & . & . \\
\hline & & $\%$ change & -73.49 & -73.61 & -73.20 & & \\
\hline & \multirow{3}{*}{$\begin{array}{c}\text { AST } \\
\text { (IU/L) }\end{array}$} & Alive & 35.10 & 44.83 & 46.00 & 43.83 & 46.50 \\
\hline & & Dead & 15.20 & 15.32 & 16.07 & . & . \\
\hline & & $\%$ change & -56.69 & -65.82 & -65.06 & . & . \\
\hline & \multirow{3}{*}{$\begin{array}{c}\text { ALP } \\
\text { (IU/L) }\end{array}$} & Alive & 82.66 & 80.66 & 74.35 & 77.50 & 71.50 \\
\hline & & Dead & 19.10 & 19.20 & 19.27 & . & . \\
\hline & & $\%$ change & -76.89 & -76.19 & -74.08 & & \\
\hline & \multirow{3}{*}{$\begin{array}{l}\text { GGT } \\
\text { (IU/L) }\end{array}$} & Alive & 13.26 & 13.48 & 13.33 & 13.40 & 12.33 \\
\hline & & Dead & 6.01 & 6.22 & 7.12 & . & . \\
\hline & & $\%$ change & -54.67 & -53.85 & -46.58 & . & . \\
\hline \multirow{12}{*}{ Shami } & \multirow{3}{*}{$\begin{array}{c}\text { ALT } \\
(\mathrm{IU} / \mathrm{L})\end{array}$} & Alive & 53.72 & 56.89 & 66.00 & 68.92 & 66.66 \\
\hline & & Dead & 15.01 & 15.05 & 16.03 & 16.60 & 17.40 \\
\hline & & $\%$ change & -72.05 & -73.54 & -75.71 & -75.91 & -73.89 \\
\hline & \multirow{3}{*}{$\begin{array}{c}\text { AST } \\
\text { (IU/L) }\end{array}$} & Alive & 34.33 & 37.92 & 39.60 & 46.55 & 56.32 \\
\hline & & Dead & 12.20 & 12.40 & 12.60 & 12.76 & 13.20 \\
\hline & & $\%$ change & -64.46 & -67.29 & -68.18 & -72.58 & -76.56 \\
\hline & \multirow{3}{*}{$\begin{array}{c}\text { ALP } \\
\text { (IU/L) }\end{array}$} & Alive & 84.44 & 82.66 & 82.88 & 82.31 & 74.77 \\
\hline & & Dead & 20.01 & 20.20 & 20.35 & 21.01 & 21.40 \\
\hline & & $\%$ change & -76.30 & -75.56 & -74.75 & -74.47 & -71.37 \\
\hline & \multirow{3}{*}{$\begin{array}{c}\text { GGT } \\
(\mathrm{IU} / \mathrm{L})\end{array}$} & Alive & 13.75 & 12.92 & 12.61 & 13.23 & 11.90 \\
\hline & & Dead & 6.09 & 6.13 & 6.17 & 7.03 & 7.15 \\
\hline & & $\%$ change & -55.70 & -52.55 & -52.24 & -47.58 & -39.91 \\
\hline
\end{tabular}

ALT: alanine transferase; AST: aspartate transferase; ALP: alkaline phosphatase; GGT: gamma glutamyl transferase 


\section{Urea and Creatinine concentrations of Baladi and Shami kids during neonatal period}

The data presented in table 9 revealed that goat breed, neonatal period affected urea concentration significantly increases in Baladi kids as compared to Shami kids, while, they exhibited insignificant effect on creatinine level. Concerning to the experimental period in present study that plasma urea had significantly decreased at day 0 and 7 th day of life while it showed significant increase at 14th, 21th and 28th day, while creatinine significantly increased at day 0 and 7th as compared to the 14th and 12th day and continued to decline until the 28th day of life (Abdolvahabi, 2016) concluded that plasma concentrations of urea and creatinine were below the adult reference intervals (ARI), the decrease in plasma urea concentrations during the first 10 days of life in neonates was in accordance with previous studies by Herosimczyk et al. (2011) and Antunović et al. (2017). The decrease in plasma creatinine concentrations after $14^{\text {th }}$ day of birth is may be associated with increased glomerular filtration rate (Herosimczyk et al., 2011). As shown in table 10 generally all kidney functions parameters (urea and creatinine) for dead kids in both breeds were insignificantly decreased as compared to alive ones, the percentages of urea within the 14 days post-partum were between (-47.06:$54.06 \%)$ in and (-46.93: $-52.97 \%)$ in urea for Baladi and Shami kids respectively through the same period, then increased within the following two weeks to reach $(-56.36$ : $-57.48 \%)$ in Shami kids, while percentages of creatinine within the 14 days post-partum were between $(-10.31:-35.50 \%)$, for Baladi dead kids and the percentage in Shami dead kids were (27.14: $-34.59 \%)$, then decreased (-13.33: $-28.93 \%)$. The reductions of kidney functions (urea and creatinine) were higher in Shami kids that might give reason to higher mortality rates in this breed.

Table 9. Evaluating kidney function of Baladi and Shami kids during the first month of post-partum

\begin{tabular}{|c|c|c|c|c|c|c|c|c|c|c|}
\hline \multirow{2}{*}{ Parameter } & \multirow{2}{*}{$\begin{array}{l}\text { Breed } \\
\text { (B) }\end{array}$} & \multicolumn{5}{|c|}{ Post-partum period (S) } & \multirow{2}{*}{ Overall } & \multicolumn{3}{|c|}{ \pm Standard error } \\
\hline & & 0 day & 7 day & 14 day & 21 day & 28 day & & B & $\mathbf{N}$ & $\mathbf{B} \times \mathbf{N}$ \\
\hline \multirow{3}{*}{$\begin{array}{l}\text { Urea } \\
(\mathrm{mg} / \mathrm{dl})\end{array}$} & Baladi & 43.82 & 40.50 & 43.38 & 44.39 & 43.68 & $43.75^{\mathrm{D}}$ & \multirow{3}{*}{$0.88^{* *}$} & \multirow{3}{*}{$1.28^{* *}$} & \multirow{3}{*}{$\begin{array}{l}1.98 \\
\text { NS }\end{array}$} \\
\hline & Shami & 35.84 & 36.05 & 40.83 & 44.28 & 45.63 & $39.93^{\mathrm{E}}$ & & & \\
\hline & Overall & $39.83^{\mathrm{BC}}$ & $38.28^{\mathrm{C}}$ & $42.10^{\mathrm{AB}}$ & $44.34^{\mathrm{A}}$ & $44.65^{\mathrm{A}}$ & & & & \\
\hline \multirow{3}{*}{$\begin{array}{l}\text { Creatinine } \\
(\mathrm{mg} / \mathrm{dl})\end{array}$} & Baladi & 1.69 & 1.45 & 1.26 & 1.35 & 1.33 & 1.42 & \multirow{3}{*}{$\begin{array}{l}0.05 \\
\text { NS }\end{array}$} & \multirow{3}{*}{$\begin{array}{c}0.07 \\
\text { NS }\end{array}$} & \multirow{3}{*}{$\begin{array}{c}0.11 \\
\text { NS }\end{array}$} \\
\hline & Shami & 1.40 & 1.59 & 1.56 & 1.59 & 1.35 & 1.50 & & & \\
\hline & Overall & 1.54 & 1.52 & 1.41 & 1.47 & 1.34 & & & & \\
\hline
\end{tabular}

B: Breed; N: Neonatal period; A, B, C: values with different letters on the same row are significantly different $(\mathrm{P}<0.05)$; D, E: values with different letters on the same column are significantly different $(\mathrm{P}<0.05)$; NS: non-significant; **: $\mathrm{P}<0.01$.

Table 10. Evaluating of kidney function and percentage change of alive and dead Baladi and Shami kids during the postnatal period

\begin{tabular}{|c|c|c|c|c|c|c|c|}
\hline \multirow{2}{*}{ Breed } & \multirow{2}{*}{ Parameter } & \multicolumn{6}{|c|}{ The neonatal period (Days) } \\
\hline & & Case & $\mathbf{0}$ & 7 & 14 & 21 & 28 \\
\hline \multirow{6}{*}{ Baladi } & \multirow{3}{*}{$\begin{array}{c}\text { Urea } \\
(\mathrm{mg} / \mathrm{dl})\end{array}$} & Alive & 43.82 & 40.50 & 43.38 & 44.39 & 43.68 \\
\hline & & Dead & 20.13 & 21.44 & 22.20 & . & . \\
\hline & & $\%$ change & -54.06 & -47.06 & -48.82 & . & . \\
\hline & \multirow{3}{*}{$\begin{array}{l}\text { Creatinine } \\
(\mathrm{mg} / \mathrm{dl})\end{array}$} & Alive & 1.69 & 1.45 & 1.26 & 1.35 & 1.33 \\
\hline & & Dead & 1.09 & 1.11 & 1.13 & . & . \\
\hline & & $\%$ change & -35.50 & -23.44 & -10.31 & . & . \\
\hline \multirow{6}{*}{ Shami } & \multirow{3}{*}{$\begin{array}{c}\text { Urea } \\
(\mathrm{mg} / \mathrm{dl})\end{array}$} & Alive & 35.84 & 36.05 & 40.83 & 44.28 & 45.63 \\
\hline & & Dead & 19.11 & 19.13 & 19.20 & 19.32 & 19.40 \\
\hline & & $\%$ change & -47.15 & -46.93 & -52.97 & -56.36 & -57.48 \\
\hline & \multirow{3}{*}{$\begin{array}{l}\text { Creatinine } \\
(\mathrm{mg} / \mathrm{dl})\end{array}$} & Alive & 1.40 & 1.59 & 1.56 & 1.59 & 1.35 \\
\hline & & Dead & 1.02 & 1.04 & 1.12 & 1.13 & 1.17 \\
\hline & & $\%$ change & -27.14 & -34.59 & -28.20 & -28.93 & -13.33 \\
\hline
\end{tabular}

\section{Physiological changes in total lipids, cholesterol and triglycerides concentration of kids during neonatal period}

The data presented in table 11 demonstrated that, Baladi kids showed insignificant increase in plasma total lipids, cholesterol and triglycerides levels when compared to Shami kids, while plasma HDL Baladi kids showed insignificant decrease level when compared to Shami kids. Concerning to post-partum days in this study showed that plasma total lipids were insignificantly higher at the first day after birth and showed reduction at the rest of post-partum period, in agreement with Öztabak and Özpinar (2006) findings that indicated that plasma total lipid concentrations of the Ewes Rearing (ER) lambs at $24 \mathrm{~h}(\mathrm{P}<0.05)$ and first week $(\mathrm{P}<0.01)$ were significantly higher than Artificial Rearing (AR) lambs. From the point of view, the high levels on total lipids after birth could be explained by the fact that fat concentration continuously increased in colostrum, Earlier, Mersmann and McNeilm (1985) reported that fatty acids in blood were the source of triglyceride synthesis in adipose tissue.

In this study plasma cholesterol and triglycerides insignificantly increase at the $7^{\text {th }}$ day till $14^{\text {th }}$ day after birth, however it showed insignificant decrease from $21^{\text {th }}$ day and reached to its highest decline at day 29th after birth. The 
results reported herein were in according to Chniter et al. (2013) who indicated that plasma cholesterol and triglycerides showed significant increasing levels in newborn lambs, this increase was attributed to that the main source was through the fatty acids in colostrum (Blum et al., 1997 and Bittrich et al., 2002). The present results showed that plasma HDL concentrations insignificant increased gradually from birth onwards. There is no previous information about the plasma lipoproteins of goats. Results in table 12 illustrated that all parameters of lipids profile analysis showed lowering in activity of these parameters in dead Baladi and Shami kids comparing with alive kids. The percentage of this reduction in Baladi dead kids within the 14 days post-partum were between (-31.79: -40.97\%) and (-29.86: $-38.60 \%)$ for Shami kids through the same period, then decreased within the following two weeks to reach (-28.96: $-32.32 \%)$ in Shami kids, while in cholesterol, triglycerides were lower in Baladi dead kids as compared with living kids within the first two weeks postpartum it were between (-41.97: -49.15\%), (-20.24: -21.36\%) for Baladi dead kids and the percentage in Shami dead kids were $(-40.02:-51.55 \%),(-19.83:-23.09 \%)$ then decreased (-7.95: -33.48), (-16.59: -19.71\%), respectively in Shami kids within the following two weeks. On the other hand, in HDL were lower in Baladi dead kids as compared with living kids within the first two weeks post-partum, it were between $(-33.54:-40.47 \%)$ for Baladi dead kids and the percentage in Shami dead kids were (-36.99: $-50.40 \%)$ then increased (-50.09: $-52.82 \%)$ respectively in Shami kids within the following two weeks. The decreasing of lipids profile measure were higher in Shami kids could be explain by higher mortality rates in this breed.

Table 11. Means of lipid parameters of Baladi and Shami kids during the first month of post-partum

\begin{tabular}{|c|c|c|c|c|c|c|c|c|c|c|}
\hline \multirow{2}{*}{ Parameter } & \multirow{2}{*}{$\begin{array}{l}\text { Breed } \\
\text { (B) }\end{array}$} & \multicolumn{5}{|c|}{ Experimental periods (S) } & \multirow{2}{*}{ Overall } & \multicolumn{3}{|c|}{ \pm Standard error } \\
\hline & & 0 day & 7 day & 14 day & 21 day & 28 day & & B & $\mathrm{N}$ & $\mathrm{B} \times \mathrm{N}$ \\
\hline \multirow{3}{*}{$\begin{array}{l}\text { Total Lipids } \\
\text { (g/l) }\end{array}$} & Baladi & 545.74 & 483.86 & 508.51 & 458.51 & 473.93 & 494.11 & \multirow{3}{*}{$\begin{array}{l}14.39 \\
\mathrm{NS}\end{array}$} & \multirow{3}{*}{$\begin{array}{l}20.77 \\
\text { NS }\end{array}$} & \multirow{3}{*}{$\begin{array}{c}32.18 \\
\text { NS }\end{array}$} \\
\hline & Shami & 506.61 & 509.69 & 456.50 & 474.58 & 453.31 & 480.14 & & & \\
\hline & Overall & 526.18 & 496.77 & 482.50 & 466.54 & 463.62 & & & & \\
\hline \multirow{3}{*}{$\begin{array}{l}\text { Cholesterol } \\
(\mathrm{g} / \mathrm{l})\end{array}$} & Baladi & $189.79^{\mathrm{bc}}$ & $216.64^{\mathrm{ab}}$ & $194.89^{\mathrm{bcd}}$ & $185.20^{\mathrm{abc}}$ & $189.50^{\mathrm{d}}$ & 195.20 & 9.79 & \multirow{3}{*}{$\begin{array}{l}14.13 \\
\text { NS }\end{array}$} & \multirow{3}{*}{$\underset{* *}{21.89}$} \\
\hline & Shami & $178.58^{\mathrm{bcd}}$ & $187.94^{\mathrm{bc}}$ & $221.90^{\mathrm{a}}$ & $162.69^{\mathrm{cd}}$ & $117.77^{\mathrm{bc}}$ & 173.78 & NS & & \\
\hline & Overall & 184.18 & 202.29 & 208.39 & 173.94 & 153.63 & & & & \\
\hline \multirow{3}{*}{$\begin{array}{l}\text { Triglycerides } \\
\text { (g/l) }\end{array}$} & Baladi & 136.33 & 137.46 & 136.80 & 136.73 & 128.20 & 135.10 & \multirow{3}{*}{$\begin{array}{c}3.49 \\
\text { NS }\end{array}$} & \multirow{3}{*}{$\begin{array}{l}5.04 \\
\text { NS }\end{array}$} & \multirow{3}{*}{$\begin{array}{c}7.80 \\
\text { NS }\end{array}$} \\
\hline & Shami & 127.37 & 128.22 & 135.37 & 124.88 & 129.82 & 129.13 & & & \\
\hline & Overall & 131.85 & 132.84 & 136.08 & 130.81 & 129.01 & & & & \\
\hline \multirow{3}{*}{$\begin{array}{l}\text { HDL } \\
(\mathrm{g} / \mathrm{l})\end{array}$} & Baladi & 180.78 & 195.22 & 202.16 & 225.81 & 245.29 & 209.85 & \multirow{3}{*}{$\begin{array}{c}14.70 \\
\text { NS }\end{array}$} & \multirow{3}{*}{$\begin{array}{c}21.22 \\
\text { NS }\end{array}$} & \multirow{3}{*}{$\begin{array}{c}32.87 \\
\text { NS }\end{array}$} \\
\hline & Shami & 206.51 & 208.93 & 262.58 & 261.37 & 278.79 & 243.63 & & & \\
\hline & Overall & 193.64 & 202.08 & 232.37 & 243.59 & 262.04 & & & & \\
\hline
\end{tabular}

B: Breed; N: Neonatal period; a, b, c, d; values in the experimental sub-groups within certain trait with different superscripts are significantly different $(\mathrm{P}<0.05)$; NS: non-significant; **: $\mathrm{P}<0.01$.

Table 12. Means of lipid parameters and percentage change of alive and dead Baladi and Shami kids during the postnatal period

\begin{tabular}{|c|c|c|c|c|c|c|c|}
\hline \multirow{2}{*}{ Breed } & \multirow{2}{*}{ Parameter } & \multicolumn{6}{|c|}{ The neonatal period (Days) } \\
\hline & & Case & 0 & 7 & 14 & 21 & 28 \\
\hline \multirow{12}{*}{ Baladi } & \multirow{3}{*}{$\begin{array}{l}\text { Total Lipids } \\
\qquad(\mathrm{g} / \mathrm{l})\end{array}$} & Alive & 545.74 & 483.86 & 508.51 & 458.51 & 473.93 \\
\hline & & Dead & 322.12 & 330.01 & 332.11 & . & . \\
\hline & & $\%$ change & -40.97 & -31.79 & -34.68 & . & . \\
\hline & \multirow{3}{*}{$\begin{array}{l}\text { Cholesterol } \\
\text { (g/l) }\end{array}$} & Alive & 189.79 & 216.64 & 194.89 & 185.20 & 189.50 \\
\hline & & Dead & 110.12 & 110.14 & 110.15 & . & . \\
\hline & & $\%$ change & -41.97 & -49.15 & -43.48 & . & . \\
\hline & \multirow{3}{*}{$\begin{array}{c}\text { Triglycerides } \\
(\mathrm{g} / \mathrm{l})\end{array}$} & Alive & 136.33 & 137.46 & 136.80 & 136.73 & 128.20 \\
\hline & & Dead & 108.05 & 108.09 & 109.11 & . & . \\
\hline & & $\%$ change & -20.74 & -21.36 & -20.24 & . & . \\
\hline & \multirow{3}{*}{$\begin{array}{l}\text { HDL } \\
(\mathrm{g} / \mathrm{l})\end{array}$} & Alive & 180.78 & 195.22 & 202.16 & 225.81 & 245.29 \\
\hline & & Dead & 120.14 & 120.24 & 120.33 & . & . \\
\hline & & $\%$ change & -33.54 & -38.40 & -40.47 & . & . \\
\hline \multirow{12}{*}{ Shami } & \multirow{3}{*}{$\begin{array}{l}\text { Total Lipids } \\
\text { (g/l) }\end{array}$} & Alive & 506.61 & 509.69 & 456.50 & 474.58 & 453.31 \\
\hline & & Dead & 311.02 & 314.12 & 320.16 & 321.15 & 322.02 \\
\hline & & $\%$ change & -38.60 & -38.37 & -29.86 & -32.32 & -28.96 \\
\hline & \multirow{3}{*}{$\begin{array}{l}\text { Cholesterol } \\
(\mathrm{g} / \mathrm{l})\end{array}$} & Alive & 178.58 & 187.94 & 221.90 & 162.69 & 117.77 \\
\hline & & Dead & 107.11 & 107.41 & 107.50 & 108.21 & 108.40 \\
\hline & & $\%$ change & -40.02 & -42.84 & -51.55 & -33.48 & -7.95 \\
\hline & \multirow{3}{*}{$\begin{array}{c}\text { Triglycerides } \\
\text { (g/l) }\end{array}$} & Alive & 127.37 & 128.22 & 135.37 & 124.88 & 129.82 \\
\hline & & Dead & 102.10 & 102.12 & 104.11 & 104.15 & 104.22 \\
\hline & & $\%$ change & -19.83 & -20.35 & -23.09 & -16.59 & -19.71 \\
\hline & \multirow{3}{*}{$\begin{array}{l}\mathrm{HDL} \\
(\mathrm{g} / \mathrm{l})\end{array}$} & Alive & 206.51 & 208.93 & 262.58 & 261.37 & 278.79 \\
\hline & & Dead & 130.11 & 130.13 & 130.22 & 130.44 & 131.51 \\
\hline & & $\%$ change & -36.99 & -37.71 & -50.40 & -50.09 & -52.82 \\
\hline
\end{tabular}




\section{Maternal behavior of Baladi and Shami does after kidding}

Dam behavior around kidding just after parturition has a major impact on kids' survival. Specific behaviors of the does (licking and grooming, helping kids with suckling) promote doe-kid recognition and a close relationship between them. The data presented in figures 1, 2 and 3 showed that overall means of does stood (sec), does started licking (sec) and first attention to kid ( $\mathrm{min}$ ) was highly significant in Shami does in comparison with Baladi does (34.70 versus 25.10)

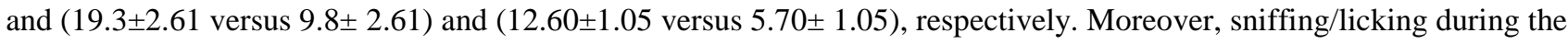
first two hours after birth (frequency) showed higher $(\mathrm{P}<0.01)$ frequency by Baladi does than Shami (193.60 versus 99.50). Within few minutes of parturition, the mother starts to lick her offspring, especially starting with its head and neck. This behavior of licking the newborn after birth optimizes the offspring's respiratory system and encourages them to stand up for suckling. The licking action of female goats is an important first stage in preparing the neonatal lamb for its new environment and life. The licking also makes the lamb clean and dry. Gul et al. (2017) reported that the average time for first licking after parturition in Awassi sheep was $138.3 \mathrm{sec}$. in single births and $154.2 \mathrm{sec}$. in twin births, while the first sniffing time intervals were $118.31 \mathrm{sec}$. and $123.6 \mathrm{sec}$, respectively.

Maternal behavior parameters showed that Baladi does stand significantly earlier than Shami does to offer care to their offspring. In addition, Baladi does significantly licked and sniffed their kids more frequently than Shami does did. These results of maternal behavior in the Baladi breed might directly relate to the reduced kid's mortality compared to Shami kids. Nowak and Poindron (2006) has made it clear that the chances of the newborn's survival and protection against external conditions will be greatly affected by the successful association between the mother and her lamb. Poindron et al. (2010) stated that the first hour after birth is critical to establishing a maternal bond towards her newborn, as pregnancy nutritional supplements can be obtained through this association regularly. for the establishment of the maternal bond towards her newborn, as nutritional supplements for a newborn can be obtained through this association regularly. The recognition by the mother of the offspring of the ewes through licking and continuous inhalation during the first postpartum hours has been described in many local hooves (Lévy et al., 2004; Lévy and Keller, 2009).

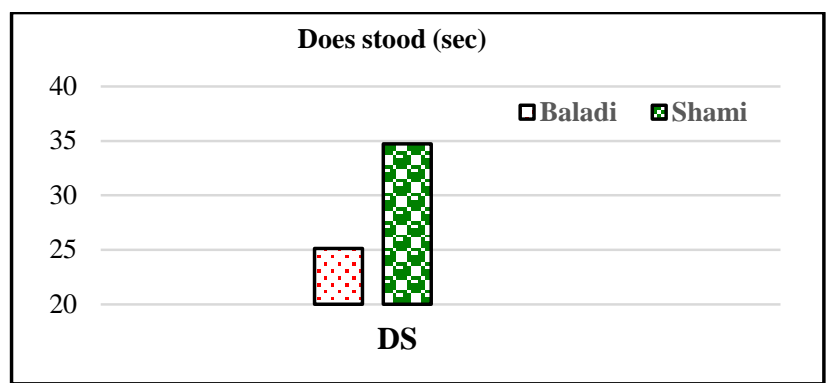

Figure 1. Overall means for Does stood (sec) of Baladi and Shami does started from kidding time. DS: Does stood $(\mathrm{sec})$

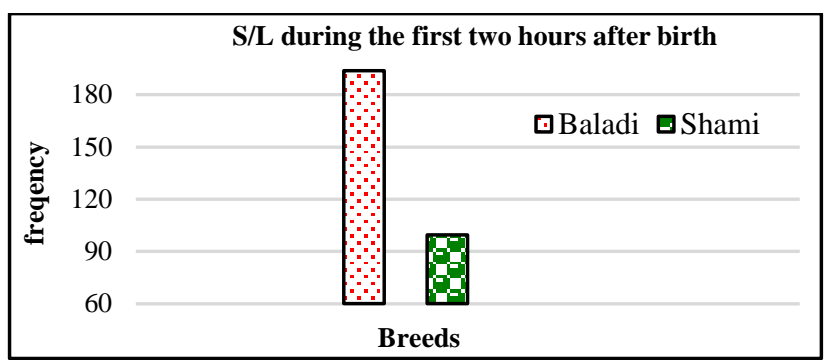

Figure 3. Overall means for maternal sniffing and licking kids during the first two hours after birth of Baladi and Shami does. S/L: Sniffing/ licking

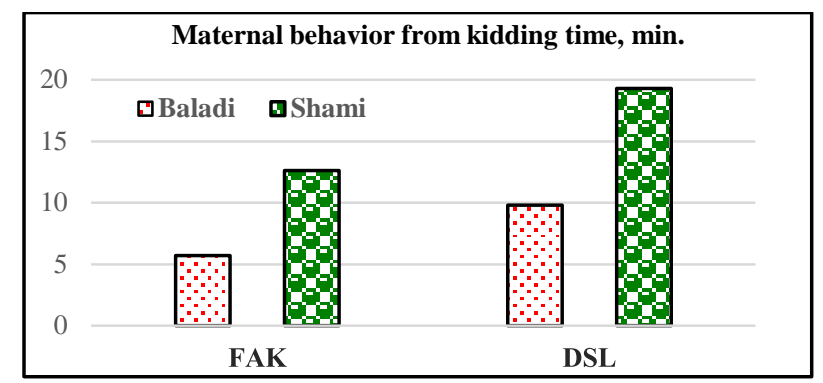

Figure 2. Overall means for first attention and does started licking kids of Baladi and Shami does. FAK: First attention to kid (min), DSL: Does started licking (min)

\section{Standing and suckling behavior of Baladi and Shami kids after delivery}

The data presented in table13 show the mean values of standing and suckling behavior parameters of newly born kids, either still alive kids or those died then after. The parameters included first standing attempt (min) and the first standing success $(\mathrm{min})$, first attempted to suckling ( $\mathrm{min}$ ), kids starting suckling $(\mathrm{min})$, suckling frequency during the first two hours after birth (bouts). For alive kids, all parameters were affected significantly $(\mathrm{P}<0.01)$ by breed differences except duration of the first suckling ( $\mathrm{min}$ ). Baladi kids showed earlier activity toward their dams than Shami did. The duration of these parameters for Baladi and Shami kids respectively were 20.40 versus 30.30 min for the first standing attempt, 35.10 versus $46.40 \mathrm{~min}$ for the first standing success, 43.2 versus $64.0 \mathrm{~min}$ for the first attempted to suckling and 50.0 versus $70.0 \mathrm{~min}$ for kids starting suckling. However, suckling frequency during the first two hours after birth was greater $(\mathrm{P}<0.01)$ in Shami kids $(16.6$ bouts) than in Baladi kids $(12.25$ bouts). This might due to higher number of kids seeking and competing one mother. 
Everett et al. (2005) explained that viability rate of offspring depends on suckling the first milk, colostrum. For this purpose, offspring must stand up as soon as possible. The maternal behavior of the dam plays an important role in the behavioral interactions that occur between ewe and their offspring during the first few hours following birth and ultimately ends in the suckling behavior. Ceyhan et al. (2012) reported that mothers must help their offspring to stand up and reach the udder. Most recently, Gul et al. (2017) studied the behavioral characteristics of Awassi sheep and their offspring during the first hour after parturition and found that all lambs tried to stand up for suckling and succeeded during the first hour after they were born. Earlier, Dwyer (2003) reported the first attempt for standing up time as 5.7 min, and successful standing-up time was $19.04 \mathrm{~min}$. Abdul-Rahman and Yaro (2010) reported that the standing-up times ranged from 4 to $79 \mathrm{~min}$, averaging $24.01 \mathrm{~min}$, in Djallonke lambs. Madani et al. (2013) found the successful standing-up time after birth to be about $23.45 \mathrm{~min}$ in sheep.

Dwyer (2003) reported an average successful suckling time of 19.04 min, while Abdul-Rahman and Yaro (2010) found a mean time for first successful suckling of 35.35 min (a range from 5 to 105 min) in Djallonke lambs. Madani et al. (2013) Noticed that standing after delivery and reaching the udder occurred within $32.7 \pm 19.02$ minutes and suckling started within 41.9 min in Djellal lambs. Mahboub et al. (2013) indicated that lambs and kids born in well-fed dams with a high level of concentration (135\%) exhibited more suckling behavior during the first week after birth and spent significantly more time licking their offspring and preparing them.

Total time of suckling during the first two hours after birth (min) was higher $(\mathrm{P}<0.01)$ in Baladi kids $(536.0$ min) than in Shami ones (38.5 min). Suckling and high milk production plays an important role in establishing a good relationship between dams and their offsprings (Nowak et al., 1997; Mahboub et al., 2013).

Kids died then after in both Baladi and Shami breeds were born very weak (physically); due to their lower birth weight; took very long time seeking their mothers and starting suckling when compared to those still alive to the end of study (Table 7). The dead Shami kids took a highly significant $(\mathrm{P}<0.01)$ longer time to stand and suckle than the dead Baladi kids. First standing attempt, the first standing success, first attempted to suckle and kids starting suckling in Baladi and Shami kids that died then after were 35.15 versus $44.14 \mathrm{~min}, 43.11$ versus $51.09 \mathrm{~min}, 61.11$ versus 73.14 and 69.10 versus 80.14, respectively. During the first two hours, those Shami kids took less time standing (26.63 min) and duration of the first suckling (1.19 min) when compared with Baladi kids (33.13 and 1.40 min, respectively). Again, this might explain partially the higher kids' mortality in Shami goats.

The differences in does and their kid's behavioral activities after birth could explain partially the higher kids' mortality in Shami goats.

Table 13. Means for standing and suckling behavior of Baladi and Shami kids after birth

\begin{tabular}{|c|c|c|c|c|c|c|c|c|}
\hline \multirow[b]{2}{*}{ Indices } & \multicolumn{4}{|c|}{ Still alive kids } & \multicolumn{4}{|c|}{ Later dead kids } \\
\hline & $\begin{array}{c}\text { Baladi } \\
\text { kids }\end{array}$ & $\begin{array}{c}\text { Shami } \\
\text { kids }\end{array}$ & $\begin{array}{c}\text { Overall } \\
\text { Mean }\end{array}$ & $\begin{array}{l}\text { Breed } \\
\pm \mathrm{SE}\end{array}$ & $\begin{array}{c}\text { Baladi } \\
\text { kids }\end{array}$ & $\begin{array}{l}\text { Shami } \\
\text { kids }\end{array}$ & $\begin{array}{l}\text { Overall } \\
\text { Mean }\end{array}$ & $\begin{array}{c}\text { Breed } \\
\pm \text { SE }\end{array}$ \\
\hline First standing attempt (min) & $20.40^{\mathrm{b}}$ & $30.30^{\mathrm{a}}$ & 25.35 & $1.15^{* *}$ & $35.15^{\mathrm{B}}$ & $44.12^{\mathrm{A}}$ & 39.63 & $1.35^{* *}$ \\
\hline First standing success (min) & $35.10^{\mathrm{b}}$ & $46.40^{\mathrm{a}}$ & 40.75 & $2.34^{* *}$ & $43.11^{\mathrm{B}}$ & $51.09^{\mathrm{A}}$ & 47.50 & $2.38^{* *}$ \\
\hline $\begin{array}{l}\text { standing time during the first two hours after } \\
\text { birth (min) }\end{array}$ & $71.60^{\mathrm{a}}$ & $51.80^{\mathrm{b}}$ & 61.70 & $2.79^{* *}$ & $33.13^{\mathrm{A}}$ & $20.14^{\mathrm{B}}$ & 26.63 & $1.22^{* *}$ \\
\hline First attempted to suckling ( $\mathrm{min}$ ) & $43.20^{\mathrm{b}}$ & $64.0^{\mathrm{a}}$ & 53.60 & $3.02^{* *}$ & $61.11^{\mathrm{B}}$ & $73.14^{\mathrm{A}}$ & 67.12 & $3.16^{* *}$ \\
\hline Kids starting suckling(min) & $50.00^{\mathrm{b}}$ & $70.00^{\mathrm{a}}$ & 60.00 & $4.81^{* *}$ & $69.10^{\mathrm{B}}$ & $80.14^{\mathrm{A}}$ & 74.62 & $4.88^{* *}$ \\
\hline Duration of first suckling (min) & 5.70 & 4.60 & 5.15 & $0.71^{\mathrm{NS}}$ & 1.40 & 1.19 & 1.29 & $0.26^{\mathrm{NS}}$ \\
\hline $\begin{array}{l}\text { Total time of suckling during the first two hours } \\
\text { after birth (min) }\end{array}$ & $52.00^{\mathrm{a}}$ & $38.50^{\mathrm{b}}$ & 45.25 & $2.02^{* *}$ & $10.13^{\mathrm{A}}$ & $8.21^{\mathrm{B}}$ & 9.17 & $0.77^{* *}$ \\
\hline $\begin{array}{l}\text { Suckling frequency during the first two hours } \\
\text { after birth (bouts) }\end{array}$ & $12.20^{\mathrm{b}}$ & $16.60^{\mathrm{a}}$ & 14.40 & $0.88^{* *}$ & $5.20^{\mathrm{B}}$ & $7.11^{\mathrm{A}}$ & 6.15 & $0.70^{* *}$ \\
\hline
\end{tabular}

SE: standard Error; a, b: values with different letters on the same row differ at $(\mathrm{P}<0.05)$; A, B: values with different letters on the same row differ at $(\mathrm{P}<0.05)$; NS: non-significant; $* * \mathrm{P}<0.01$

\section{CONCLUSION}

In the present study, kids' mortality occurred in Baladi during the first two weeks, while continued in Shami kids all over the first month with different percentage per week. Also, it was apparent that Shami female kids had the highest birth weight, growth rate and final LBW. Moreover, Baladi male kids that could stay alive compensated the lower birth weight by high relative growth rate and attained the same final LBW as Shami males. Also, that decreasing in liver enzymes (AST, ALT, ALP) activities, kidney functions (urea and creatinine) and lipids profile analysis were higher in Shami kids that might give reason to higher mortality rates in this breed. 
So that, Baladi does and their kids overpowered their Shami counterparts in some biochemical parameters and some behavioral characteristics, which might enhance kids' viability and reduce mortality rates. Moreover, the number of weak kids with lower body weight was more pronounced in Shami goats that led to higher mortality rate.

\section{DECLARATIONS}

\section{Acknowledgments}

The authors are thankful to Ras Sudr Station Staff for contributed during sample collection.

\section{Competing interests}

The authors declare that they have no conflict of interest with respect to the research, authorship, and/or publications of this article. The authors declare that they have no competing interests.

\section{Author's contribution}

Dr. N. H. Ibrahim designed the experiment, helped in field study, tabulation of experimental data, statistical analysis, article writing and revision. Dr. M. T. Badawy facilitates the field study, manuscript writing and revision. Dr. I. A. Zakzouk helped in field study, collected data, tabulation of experimental data and manuscript writing. Dr. F. E. Younis helped in field study. All authors have read and approved the final manuscript.

\section{REFERENCES}

Abdolvahabi S, Zaeemi M, Mohri M and Naserian AA (2016). Age related changes in serum biochemical profile of Saanen goat kids during the first three months of life. Revue de Médecine Vétérinaire, 167 (3):106-112. Available at: http://www.revmedvet.com/2016/RMV167_106_112.pdf

Abdul-Rahman II and Yaro M (2010). The effect of sex, birth weight and type of birth on neonatal behavior of Djallonke sheep and West African dwarf goats. Global Veternaria,4: 409-415. Available at: https://www.idosi.org/gv/gv4(4) 10/16.pdf

Aganga AA, Omphile UJ, Chabo RG, Kgosimore M and Mochankana M (2005). Goat production under traditional management in Gaborone agricultural region in Botswana. Journal of Animal and Veterinary Advances, 4(5): 515-566. DOI: https://doi:javaa.2005.515.519

Al-Seaf AM and Al-Harbi KB (2012). Variability of disease resistance, haematological parameters and lymphocyte proliferation in two goat breeds and their F1 and F2 crosses. International Journal of Food, Agriculture and Veterinary Sciences, 2 (1):47-53.

Antunović Z, Šperanda M and Steiner, Z (2004). The influence of age and the reproductive status to the blood indicators of the ewes. Archives Animal Breeding, 47(3): 265-273. DOI: https://doi.org/10.5194/aab-47-265-2004

Antunović Z, Šperanda M, Novoselec J, Đidara M, Mioč B, Klir Ž and Samac D (2017). Blood metabolic profile and acid-base balance of dairy goats and their kids during lactation. Veterinarski Arhiv, 87(1): 43-55. Available at: https://hrcak.srce.hr/178520

Ashour G, Neama A A, Dessouki SM and Shihab OH (2015). Blood hematology, metabolites and hormones in newborn sheep and goat from birth to weaning. International Journal of Advanced Research, 3 (7): 1377-1386.

Association of Official Analytical Chemists (1990). Official Methods of Analysis, 13th Ed. Washington, D. C.

Awemu EM, Nwakalor LN and Abubakar BY (1999). Environmental influence on preweaning mortality and reproductive performance of Red Sokoto does. Small Ruminant Research, 34. 161-165. DOI: https://10.1016/S0921-4488(99)00058-9

Awemu EM, Nwakalo LN and Abubakar BY (2002). The biological productivity of the Yankasa sheep and the Red Sakoto goat in Nigeria. Dept. of Animal Science, University of Nigeria, Nsukka, Nigeria.

Bittrich S, Morel C, Philipona C, Zbinden Y, Hammon HM and Blum JW (2002). Physiological traits in preterm calves during their first week of life. Journal of Animal Physiology and Animal Nutrition, 86: 185-198. DOI: https://10.1046/j.14390396.2002.00372.x

Blum JW, Hadorn U, Sallmann HP and Schuep W (1997). Delaying colostrum intake by one day impairs the plasma lipid, essential fatty acid, carotene, retinol and tocopherol status in the neonatal calves. The Journal of Nutrition, 127: 2024-2029. DOI: https://10.1093/jn/127.10.2024

Browning JR and Leite $\neg$ Browning ML (2011). Birth to weaning kid traits from a complete diallel of Boer, Kiko, and Spanish meat goat breeds semi intensively managed on humid subtropical pasture. Journal of Animal Science, 89: 2696 - 22707. DOI: https://10.2527/jas.2011-3865

Bushara M, Idris DM, Elemam AO, Abdelhadi MB, Muna OM, Ahmed MM, Abu Nikhiala AM and Alimon AR (2013). Productivity of taggar goats as affected by sex of kids and litter size. Basic Research Journal of Agricultural Science and Review, 2(5): 116121. DOI: https://10.14196/aa.v2i5.765

Central Agency for Public Mobilization and Statistics (2016). Central Agency for Public Mobilization and Statistics. Available at: http://www.capmas.gov.eg/Pages/StaticPages.aspx?page_id=5035.

Ceyhan A, Sezenler T, Yüksel MA and Yıldırır M (2012). Maternal and lamb behavior of the Karacabey Merino ewes at pre- and post-parturition. Research Opinions in Animal \& Veterinary Sciences, 2: 402-408. Available at: https://www.researchgate.net/publication/255982904 
Chniter M, Hammadi M, Khorchani T, Sassi MB, Hamouda MB and Nowak R (2013). Aspects of neonatal physiology have an influence on lambs' early growth and survival in prolific D'man sheep. Small Ruminant Research, 111(1): 162-170. DOI: https://10.1016/j.smallrumres.2012.10.004

Daramola JO, Adeloye AA, Fatoba TA and Soladoye AO (2005). Haematological and biochemical parameters of West African Dwarf goats. Livestock Research for Rural Development, 17(8). Available at: https://www.lrrd.cipav.org.co/lrrd17/8/dara17095.htm

Debele G, Duguma M and Hundessa F (2011). Effect of different factors on mortality rate of Arsi-Bale kids in mid rift valley of Ethiopia. Global Veterinaria, 6(1):56-60.

Donkin EF and Boyazoglu P (2004). Diseases and mortality of goat kids in a South African milk goat herd. South African Journal of Animal Science, 34: 220-223. https://www.researchgate.net/publication/242254435

Duncan DB (1955). Multiple range and multiple F tests. Biometrics, 11(1): 1-42. DOI: https://10.2307/3001478

Dwyer CM (2009). The ethology of domestic animals. In: Jensen, P. (ed.). The behavior of sheep and goats. CAB International, London UK. pp. 161-176. DOI: https://10.1079/9781786391650.0199

Dwyer CM (2003). Behavioral development in the neonatal lamb: effect of maternal and birth-related factors. Theriogenology, 59: 1027-1050. DOI: https://10.1016/s0093-691x(02)01137-8

ELimam ME, Mustafa AM and Mohamed KA (2007). Characterization of the Tagger goats in Eldelang area in the Nuba mountain in South Kordofan., Sudan. Conference. on genetic resources of the Sudanese livestock wealth. Khartoum.

EL-Tonsy MM, Ibrahim NH, Farid NM, Badawy MT and Essa AE (2018). Scrutinizing the Effect of some Physiological and Histopathological Factors on Kids Mortality of Shami and Baldi Goat during Neonatal Period. Journal of Animal and Poultry Production, 9 (2): 123 - 134. DOI: https://10.21608/jappmu.2018.38943

Elitok B (2012). Reference values for hematological and biochemical parameters in Saanen goats breeding in Afyonkarahisar province. Kocatepe Veteriner Dergisi, 5(1):7-11. Available at: https://acikerisim.aku.edu.tr/xmlui/handle/11630/2301

Everett-Hincks JM, Lopez-Vilalobos N, Blair HT and Stafford KJ (2005). The effect of maternal behavior score on Iamb and litter survival Livestock Production Science, 93: 51 - 61. DOI: https://10.1016/j.livprodsci.2004.11.006

Food and Agriculture Organization Corporate Statistical Database (2012). Characterization and value addition to local breeds and their products in the near east and north Africa, FAO Animal production and health paper No. 19-21 November, Rabat, Morocco.

Galal S, Abdel-Rasoul F, Anous MR and Shaat I (2005). On-station characterization of small ruminant breeds in Egypt. In: L. In iguez (ed.), Characterization of Small Ruminant Breeds in West Asia and North Africa. Small Ruminant Research, 60: 75-81.

Girma D, Misgana D and Feyisa H (2011). Effect of Different Factors on Mortality Rate of Arsi- Bale Kids in Mid Rift Valley of Ethiopia. Adami-Tullu Agricultural Research Center. Global Veterinaria, 6 (1): 56-60.

Gubartalla KE, Abu Nam A and EL Khidir O (2002). Some observations on performance of first kidding Sudanese Nubian goats. Sudan Journal of Animal Production, 15:43-54.

Gul $\mathrm{F}$ and Efeoglu $\mathrm{H}$ (2017). $\mathrm{ZnO}$ and $\mathrm{ZnO} 1-\mathrm{x}$ based thin film memristors: The effects of oxygen deficiency and thickness in resistive switching behavior. Ceramics International, 43(14): 10770-10775. DOI: https://10.1016/j.ceramint.2017.05.090

Gwaze FR, Chimonyo M and Dzama K (2012). Effect of season and age on blood minerals, liver enzyme levels, and faecal egg counts in Nguni goats of South Africa. Czech Journal of Animal Science, 57(10): 443-453. Doi: https://10.17221/6345-CJAS

Hailu D, Mieso G, Nigatu A, Fufa D and Gamada D (2006). The effect of Environmental factors on pre-weaning survival rate of Borana and Arsi-bale Kids. Small Ruminant Research, 66:291-294. DOI: https://10.1016/j. smallrumres.2005.09.024

Hanford KJ, Van Vleck LD and Snowder GD (2006). Estimates of genetic parameters and genetic trend for reproduction, weight, and wool characteristics of Polypay sheep. Livestock Science, 102:72-82. Doi: https://10.1016/j.livsci.2005.11.002

Herosimczyk A, Lepczyński A, Dratwa-Chałupnik A, Kurpińska A, Klonowska A and Skrzypczak WF (2011). Age-related changes of selected blood biochemical indicators in dairy calves during their first week of life. Folia Biologica (Krakow): 59, 25- 30. Doi: https://10.3409/fb59_1-2.25-30

Ingo $\mathrm{H}$ (2002). Analysis of survival curves in seasonally mated pastoral goat herds in northern Kenya using logistic regression techniques. Journal of Arid Environments, 50: 621-640. Doi: https://10.1006/jare.2001.0919

Kaymakci M (2013). İleri Keçi Yetiştiriciliği (Advanced Goat Breeding). ISBN 978-605-85998-0-2. Bornova, İzmir.

Kearl, LC (1982). Nutrient requirements of ruminants in developing countries. International Feedstuffs Institute, Utah, U.S.A.

Khalil MH, Badawy MT, Badr MI, Abdel Hamid AA and Wahba IA (2018). Changes in some Hemato-Biochemical Parameters and Hormonal Aspects in Baladi and Shami Kids in Relation to Their Mortality Rates during the Postnatal Period. Journal of Animal and Poultry Production, 9 (8): 357 - 365. DOI: https://10.21608/jappmu.2018.41139

Khazaal K (2009). Comparison of performance of Shami (Damascus) and Saanen goats raised under similar environmental condition in Lebanon. Option Mediterranean's, No 85. Kids. Indian Journal Animal Science,72: 161-164.

Lévy F and Keller M (2009). Olfactory mediation of maternal behavior in selected mammalian species. Behavioral Brain Research, 200(2): 336-345. DOI: https://10.1016/j.bbr.2008.12.017

Lévy F, Keller M and Poindron P (2004). Olfactory regulation of maternal behavior in mammals. Hormones and Behavior, 46: 284 302. DOI: https://10.1016/j.yhbeh.2004.02.005

Madani T, Allouche L, Saffidine N, Kaouane N, Belkasmi F and Semara L (2013). Maternal and neonatal behaviors of Ouled Djellal sheep breed and their effects on production parameters (Short Communication). Small Ruminant Research, 114: 46-50. DOI: https://10.1016/j.smallrumres.2013.06.003

Madibela OR, Mosimanyana BM, Boitumelo WS and Pelaelo TD (2002). Effect of supplementation on reproduction of wet season kidding Tswana goats. South African Journal of Animal Science, 32(1): 1-22. DOI: https://10.4314/sajas. v32i1.3786

Mahboub HD, Ramadan SG, Helal MA and Aziz EA (2013). Effect of maternal feeding in late pregnancy on behavior and performance of Egyptian goat and sheep and their offspring. Global Veterinary, 11: 168-176. DOI: https://10.5829/idosi.gv.2013.11.2.74152 
Martínez M, Otal J, Ramírez A, Hevia ML and Quiles A (2009). Variability in the behavior of kids born of primiparous goats during the first hour after parturition: Effect of the type of parturition, sex, duration of birth, and maternal behavior. Journal of Animal Science, 87:1772 -1777. DOI: https://10.2527/jas.2008-1311

Mersmann HJ and MacNeil MD (1985). Relationship of plasma lipid concentrations to fat deposition in pigs. Journal of Animal Science, 61: 122-128. DOI: https://10.2527/jas1985.611122x

Mtenga LA, Owen E, Muhikambele VR, Kifaro GC, Sendalo DC, Massawe, NF, Kiango SM and Nkungu DR (1992). Growth and partition of fat depots in male British Saanen goats. Proceedings of the Third Biennial Conference of the African Small Ruminant Research Network, UICC, Kampala, Uganda, 5-9 December 1992.

Mtenga LA, Kifaro GC and Berhanu B (2008). Studies on factors affecting reproductive performance and mortality rates of Small East African goats and their crosses. Small Ruminant Research, 43: 1- 5.

Njidda AA, Shuai'bu AA and Isidahomen CE (2014). Haematological and serum biochemical indices of sheep in semi-arid environment of northern Nigeria. Global Journal of Science Frontier Research. D Agriculture and Veterinary, 14(2): 49-53.

Nowak R, Murphy TM, Lindsay DR, Alster P, Andersson R and Uvnas-Moberg K (1997). Development of a preferential relationship with the mother by the newborn lamb: importance of the sucking activity. Physiology \& Behavior, 62: 681-688. DOI: https://10.1016/s0031-9384(97)00079-6

Nowak R and Poindron P (2006). From birth to colostrum: Early steps leading to lamb survival. Reproduction Nutrition Development, 46:431-446. DOI: https://10.1051/rnd:2006023

Nowak R, Keller M, Val-Laillet D and Lévy F (2007). Perinatal visceral events and brain mechanisms involved in the development of mother young bonding in sheep. Hormones and Behavior, 52: 92-98. DOI: https://10.1016/j.yhbeh.2007.03.021.

Nowak R, Porter RH, Levy F, Orgeur P and Schaal B (2000). Role of mother-young interactions in the survival of offspring in domestic mammals. Reviews of Reproduction, 5:153-163. DOI: https://10.1530/ror.0.0050153

Öztabak K and Özpinar A (2006). Growth performance and metabolic profile of Chios lambs prevented from colostrum intake and artificially reared on a calf milk replacement. Turkish Journal of Veterinary and Animal Sciences, 30: 319-324.

Petros A, Kassaye A and Berhanu S (2014). Pre-weaning kid mortality in Adamitulu Jedokombolcha District, Mid Rift Valley, Ethiopia. Journal of Veterinary Medicine and Animal Health, 6(1): 1-6.

Piccione G, Casella S, Pennisis P, Giannetto C, Costa A and Caola G (2010). Monitoring of physiological and blood parameters during perinatal and neonatal period in calves. Arquivo Brasileiro de Medicina Veterinária e Zootecnia, 62: 1-12. DOI: https://10.1590/S0102-09352010000100001

Poindron P, Keller M and Lévy F (2007). Maternal responsiveness and maternal selectivity in domestic sheep and goats: the two facets of maternal attachment. Developmental Psychobiology, 49: 54-70. DOI: https://10.1002/dev.20192

Poindron P, Otal J, Ferreira G, Keller M, Guesdon V, Nowak R and Lévy F (2010). Amniotic fluid is important for the maintenance of maternal responsiveness and the establishment of maternal selectivity in sheep. Animal, 4: 2057-2064. DOI: https://10.1017/S1751731110001126

Portolano B, Todaro M, Finocchiaro R and van Kaam JH (2002). Estimation of the genetic and phenotypic variance of several growth traits of the Sicilian Girgentana goat. Small Ruminant Research, 45: 247-253. DOI: https://10.1016/S0921-4488(02)00161-X

SAS (2004). Statistical Analysis System, STAT/ user's guide, Release 9.1, SAS Institute, Cary NC. USA.

Smith MC and Shermand M (2009). Goat Medicine, (2nd ed), Willy-Blackwell Publication, UK.

Snyman MA (2010). Factors affecting pre-weaning kid mortality in South African Angora goats. South African Journal of Animal Science, 40:54-64. DOI: https://10.4314/sajas.v40i1.54128

Steve DC and Marco FB (2001). Birthdate, mass and survival in mountain goat kids: effect of maternal characteristics and forage quality. Oecologia, 127: 230-238. DOI: https://10.1007/s004420000584

Tambuwal FM, Agaie BM and Bangana A (2002). Hematological and Biochemical Values of Apparently Healthy Red Sokoto Goat s. Proceeding of 27th Annual Conference of Nigerian Society of Animal production (NSAP), March 17-21, FUTA, Akure, Nigeria. pp. 50-53.

Tibbo M, Jibril Y, Woldemeskel M, Dawo F, Argaw K and Rege JEO (2008). Serum enzyme levels and influencing factors in three indigenous Ethiopian goats breeds. Tropical Animal Health and Production, 40: 657-666. DOI: https://10.1007/s11250-0089145-2. 